\title{
Microcracking in Composite Laminates: Simulation of Crack-Induced Ultrasound Attenuation
}

\author{
C.A.C. Leckey, M.D. Rogge, and F.R. Parker \\ Nondestructive Evaluation Sciences Branch, NASA Langley Research Center, Hampton, \\ VA 23681
}

\begin{abstract}
Microcracking in composite laminates is a known precursor to the growth of inter-ply delaminations and larger scale damage. Microcracking can lead to the attenuation of ultrasonic waves due to the crack-induced scattering. 3D elastodynamic finite integration technique (EFIT) has been implemented to explore the scattering of ultrasonic waves due to microcracks in anisotropic composite laminates. X-ray microfocus computed tomography data was directly input into the EFIT simulation for these purposes. The validated anisotropic 3D EFIT code is shown to be a useful tool for exploring the complex multiple-scattering which arises from extensive microcracking.
\end{abstract}

Keywords: Finite integration technique, EFIT, Ultrasound Simulation, Composite, Anisotropic PACS: $43.35 . \mathrm{Zc}, 43.20 . \mathrm{Gp}$, 43.35.Cg

\section{INTRODUCTION}

The use of composite materials in the aerospace industry has increased in recent years. NASA is currently involved in research related to the use of composites in space applications, such as the Composite Crew Module [1], as well as ongoing research on the use of advanced composites for aviation. The large-scale use of composites for aerospace vehicles/components leads to a requirement for accurate damage detection methods that are appropriate for the damage types that can occur in composite materials. Repeated or close proximity impact-induced delamination damage that is just below the "barely visible" limit is an example of a damage situation which may be a unique concern for composites in aerospace applications. Microcracking of the matrix material is another known damage type that can occur in composites, and is a known precursor to larger scale damage and delamination growth [2,3]. Ultrasonic inspection methods are currently among the most widely used techniques implemented for aircraft maintenance, and are promising for the detection of the early onset of damage in composites in the form of microcracking $[4,5]$.

Modeling of ultrasonic wave behavior and interaction with damage can guide the development of quantitative damage detection and characterization techniques for nondestructive evaluation (NDE) and structural health monitoring (SHM), and can provide an understanding of complex experimental data. In previous work we used 3-dimensional 
(3D) elastodynamic finite integration technique (EFIT) simulations to explore ultrasound interaction with damage in isotropic aircraft materials [6,7]. In our current work we have expanded the 3D EFIT simulation code to model wave behavior in anisotropic composite laminates made up of orthotropic ply layers. In particular, anisotropic EFIT code was used to explore wave interaction with microcrack damage in aerospace carbon fiber reinforced polymer (CFRP) laminates.

\section{ANISOTROPIC ELASTODYNAMIC FINITE INTEGRATION TECHNIQUE}

Elastodynamic finite integration technique is a numerical method similar to staggered-grid finite difference techniques. The technique has been in use since the early 1990s with extensive foundational work reported by authors such as Fellinger, Marklein, and Schubert, among others [8-10]. The discretized EFIT velocity, $v$, and stress, $T$, equations for the case of anisotropic composite laminates, made up of orthotropic ply layers, are shown below [11]:

$$
\begin{aligned}
& \dot{v}_{1}^{(n)}(t)=\frac{2}{\Delta x\left(\rho^{(n)}+\rho^{(n+\hat{x} 1)}\right)}\left[T_{11}^{(n+\hat{x} 1)}(t)-T_{11}^{(n)}(t)+T_{12}^{(n)}(t)-T_{12}^{(n-\hat{x} 2)}(t)+T_{13}^{(n)}(t)\right. \\
& \left.-T_{13}^{(n-\hat{x} 3)}(t)\right]+f_{1} \\
& \dot{v}_{2}^{(n)}(t)=\frac{2}{\Delta x\left(\rho^{(n)}+\rho^{(n+\hat{x} 2)}\right)}\left[T_{12}^{(n)}(t)-T_{12}^{(n-\hat{x} 1)}(t)+T_{22}^{(n+\hat{x} 2)}(t)-T_{22}^{(n)}(t)+T_{23}^{(n)}(t)\right. \\
& \left.-T_{23}^{(n-\hat{x} 3)}(t)\right]+f_{2} \\
& \dot{v}_{3}^{(n)}(t)=\frac{2}{\Delta x\left(\rho^{(n)}+\rho^{(n+\hat{x} 3)}\right)}\left[T_{13}^{(n)}(t)-T_{13}^{(n-\hat{x} 1)}(t)+T_{23}^{(n)}(t)-T_{23}^{(n-\hat{x} 2)}(t)+T_{33}^{(n+\hat{x} 3)}(t)\right. \\
& \left.-T_{13}^{(n)}(t)\right]+f_{3} \\
& \dot{T}_{11}^{(n)}=\frac{1}{\Delta x}\left[C_{11}^{(n)}\left(v_{1}^{(n)}(t)-v_{1}^{(n-\hat{x} 1)}(t)\right)+C_{12}^{(n)}\left(v_{2}^{(n)}(t)-v_{2}^{(n-\hat{x} 2)}(t)\right)\right. \\
& \left.+C_{13}^{(n)}\left(v_{3}^{(n)}(t)-v_{3}^{(n-\hat{x} 3)}(t)\right)\right] \\
& \dot{T}_{22}^{(n)}=\frac{1}{\Delta x}\left[C_{21}^{(n)}\left(v_{1}^{(n)}(t)-v_{1}^{(n-\hat{x} 1)}(t)\right)+C_{22}^{(n)}\left(v_{2}^{(n)}(t)-v_{2}^{(n-\hat{x} 2)}(t)\right)\right. \\
& \left.+C_{23}^{(n)}\left(v_{3}^{(n)}(t)-v_{3}^{(n-\hat{x} 3)}(t)\right)\right] \\
& \dot{T}_{33}^{(n)}=\frac{1}{\Delta x}\left[C_{31}^{(n)}\left(v_{1}^{(n)}(t)-v_{1}^{(n-\hat{x} 1)}(t)\right)+C_{32}^{(n)}\left(v_{2}^{(n)}(t)-v_{2}^{(n-\hat{x} 2)}(t)\right)\right. \\
& \left.+C_{33}^{(n)}\left(v_{3}^{(n)}(t)-v_{3}^{(n-\hat{x} 3)}(t)\right)\right]
\end{aligned}
$$

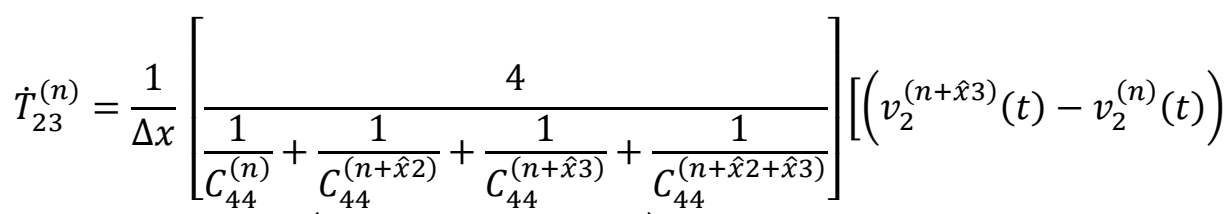

$$
\begin{aligned}
& \left.+\left(v_{3}^{(n+\hat{x} 2)}(t)-v_{3}^{(n)}(t)\right)\right]
\end{aligned}
$$




$$
\begin{gathered}
\dot{T}_{13}^{(n)}=\frac{1}{\Delta x}\left[\frac{4}{\left.\frac{1}{C_{55}^{(n)}}+\frac{1}{C_{55}^{(n+\hat{x} 1)}}+\frac{1}{C_{55}^{(n+\hat{x} 3)}}+\frac{1}{C_{55}^{(n+\hat{x} 1+\hat{x} 3)}}\right]}+\left[\left(v_{1}^{(n+\hat{x} 3)}(t)-v_{1}^{(n)}(t)\right)\right.\right. \\
\left.\quad+\left(v_{3}^{(n+\hat{x} 1)}(t)-v_{3}^{(n)}(t)\right)\right] \\
\dot{T}_{12}^{(n)}=\frac{1}{\Delta x}\left[\frac{4}{\frac{1}{C_{66}^{(n)}}+\frac{1}{C_{66}^{(n+\hat{x} 1)}}+\frac{1}{C_{66}^{(n+\hat{x} 2)}}+\frac{1}{C_{66}^{(n+\hat{x} 1+\hat{x} 2)}}}\right]\left[\left(v_{1}^{(n+\hat{x} 2)}(t)-v_{1}^{(n)}(t)\right)\right. \\
\left.\quad+\left(v_{2}^{(n+\hat{x} 1)}(t)-v_{2}^{(n)}(t)\right)\right]
\end{gathered}
$$

with:

$$
\begin{gathered}
v_{i}^{(t)}=v_{i}^{(t-1)}+\dot{v}_{i}^{(t-\Delta t / 2)} \Delta t, \quad T_{i j}^{(t+\Delta t / 2)}=T_{i j}^{(t-\Delta t / 2)}+\dot{T}_{i j}^{(t)} \Delta t \\
\Delta t \leq \frac{\Delta x}{c_{\max } \sqrt{3}}, \quad \Delta x \approx \frac{c_{\min }}{10 f_{\max }}
\end{gathered}
$$

In these equations $\Delta x$ is the spatial step size, $\Delta t$ is the temporal step size, $\rho$ is density, $C_{i j}$ are the elastic constants, the notation $\left(n+\hat{x}_{i}\right)$ means one spatial step in the $\hat{x}_{\mathrm{i}}$ direction past the current grid position, $n, c_{\min }$ and $c_{\max }$ are the minimum and maximum speeds of sound, $f_{\max }$ is the maximum frequency, and dot the notation $\dot{T}$ denotes the time derivative of the corresponding variable. We have assumed a cubic EFIT grid, such that $\Delta x_{1}=\Delta x_{2}=\Delta x_{3}$. Our implementation of these equations was validated against experimental laser doppler vibrometer wavefield data as well as against predicted dispersion curves. The EFIT simulation output was found to match closely with both experiment and theory, and details of the comparisons can be found in Ref. [12].

\section{MICROCRACK SIMULATIONS}

In order to investigate ultrasonic wave interaction with microcracks in composite laminates, we obtained a 16-ply thick IM7/5260 composite laminate panel, shown in Figure 1. The panel has a quasi-isotropic layup $[45 / 0 /-45 / 90]_{2 \mathrm{~s}}$ and is made of single fiber direction prepreg layers. The laminate specimen was previously subjected to thermalmechanical fatigue cycling [13]. The outermost ends (towards the mechanical fatigue grip holes shown in Figure 1) of the large dogbone-shaped panel were not subjected to the thermal cycling, since they were located outside of the oven. A sample cut from one of these outermost regions was found to have little to no microcracking, based on X-ray microfocus computed tomography (microCT) data. MicroCT data, with a resolution of 14.3 microns, was also taken of a sample cut towards the middle section of the dogbone specimen. This sample was found to have extensive microcracking.

The microCT data was processed using image processing edge finding techniques. The resulting image stack was then turned into a 3D microcrack 'map' (essentially a matrix with information about crack location, geometry, and size) that could be input into the EFIT simulations, see Figure 2. The crack regions were created in EFIT by implementing stress-free boundary conditions because the microCT data appears to 
contain primarily open cracks (i.e., no contact between crack edges). A primary goal of these simulations was to demonstrate that wave interaction with realistic crack geometries and distributions commonly found in anisotropic composite laminates can be modeled with EFIT.
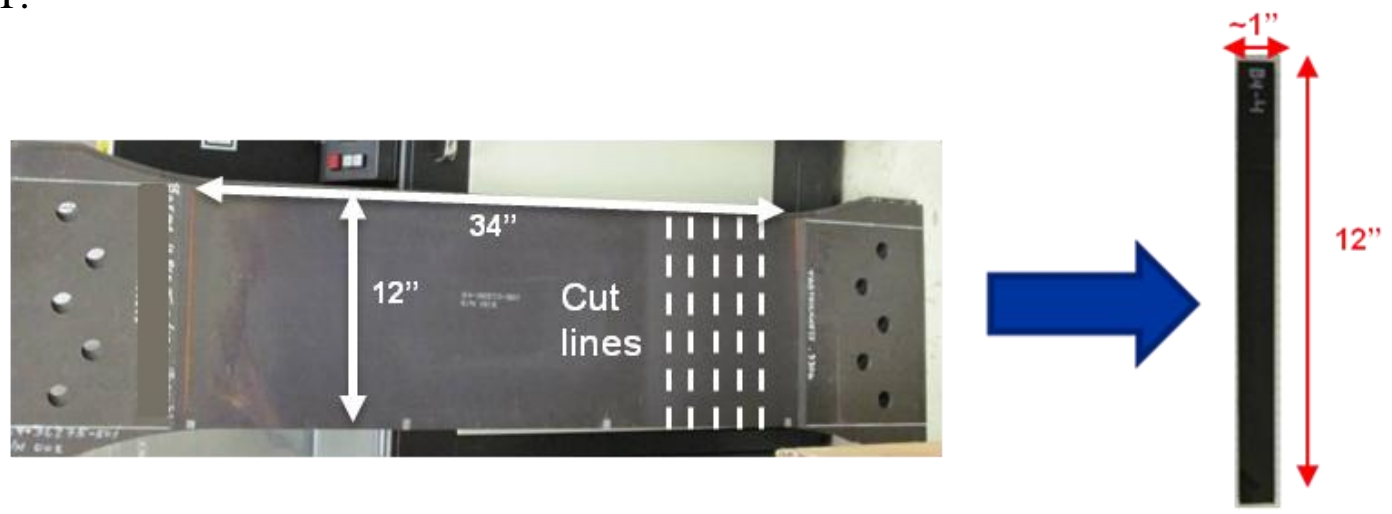

FIGURE 1. Photo of the IM7/5260 dogbone composite laminate specimen that was cut into smaller 1"x12" samples and used to obtain microCT data of composite laminate microcracking.

The ply-level material properties for IM7/5260 that were used for the EFIT simulations are shown in Table 1 , where $\rho$ is density, $\mathrm{E}$ is Young's modulus, $\mathrm{G}$ is shear modulus, and $v$ is Poisson's ratio; with $\mathrm{E}_{1}$ in the fiber direction [12,14]. The elastic constants, $\mathrm{C}_{\mathrm{ij}}$, were calculated from the properties shown in Table 1, and were rotated appropriately in order to be entered ply-by-ply into the EFIT simulations. Although the overall laminate specimen has a quasi-isotropic layup, correctly entering ply-by-ply properties is particularly pertinent since microcracks tend to run along the fiber direction in each ply layer. Thus, we have chosen to account for the anisotropic behavior of each layer in order to fully model wave interaction with the cracks.
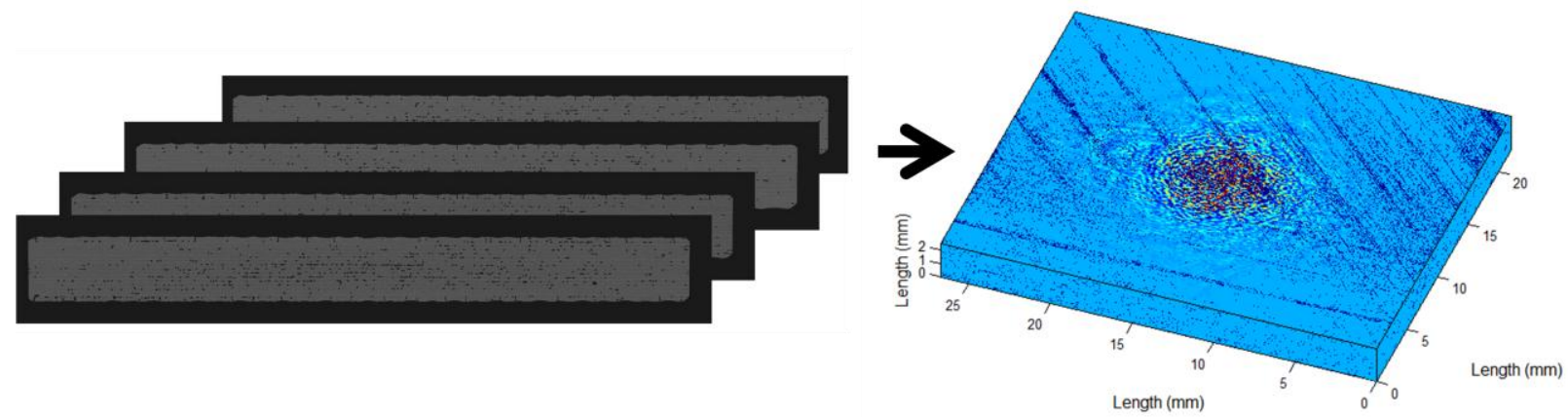

FIGURE 2. MicroCT data is processed with edge finding techniques to identify microcracks, and the resulting data is read into the 3D EFIT simulation. Left: Processed microCT microcrack data, Right: An example of 3D EFIT simulation output.

The EFIT simulations used a spatial step size of 14.3 microns, corresponding to the resolution of the microCT data and allowing for a straight-forward one-to-one mapping of the microcrack data in the EFIT simulation. The small step size was also used to ensure that there were several spatial steps per ply (11 steps per ply). A $5 \mathrm{MHz} 4$-cycle Hannwindowed sine wave was used in the simulation as the incident wave. The excitation was generated over a $1 / 8$ " circular region to correspond to the size of a contact transducer that was used to collect experimental data on the corresponding composite sample (discussed in the following section). The total simulation space size was only $26.71 \mathrm{~mm} \times 22.08 \mathrm{~mm}$ $\mathrm{x} 2.51 \mathrm{~mm}$, yet with the small step size of 14.3 microns, this required over 500 million 
simulation grid cells. Figure 3 shows 2D through-thickness slices taken from the 3D EFIT simulation output for two different locations along the simulated composite sample region, at three points in time. The images shown in the figure are intended to emphasize the importance of accounting for multiple-scattering effects in cases with such extensive microcracking. Additionally, Figure 3 demonstrates location-based variation in crack distribution and density within a very small region of a single composite sample. The 2D through-thickness data slices are taken at locations that are only $0.71 \mathrm{~mm}$ apart along the composite sample length. The location shown in the left column of the figure has microcracks that nearly form a complete delamination, and result in somewhat restricted wave propagation into the lower half of the composite laminate at that location.
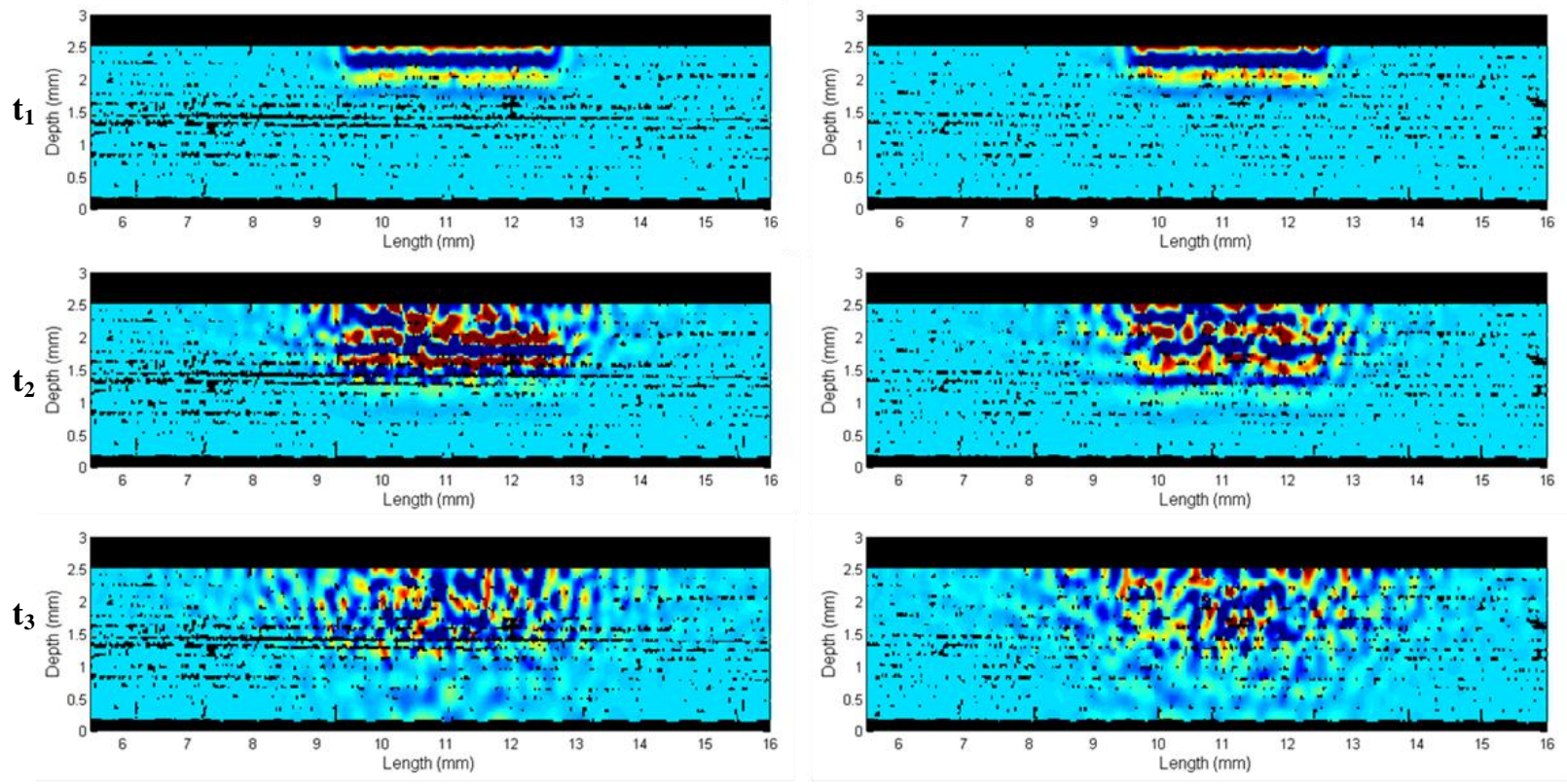

FIGURE 3. 2D through-thickness slices taken from the 3D EFIT simulation data at two different locations along the composite sample $(0.71 \mathrm{~mm}$ apart). Left column: EFIT images of through-thickness wave propagation at three points in time for vertical slice at location \#1; Right column: wave propagation at vertical slice location $\# 2$. The vertical slice shown in the left column contains a line of microcracks that nearly form a complete delamination

\section{Comparisons Between EFIT and Experiment}

MicroCT data for two different $26.71 \mathrm{~mm}$ x $22.08 \mathrm{~mm}$ x $2.512 \mathrm{~mm}$ regions along the composite microcrack sample (designated $A$ and $B$ in Figure 4) were read into EFIT in order to further explore position-based variation in crack-induced attenuation and to allow for comparisons between EFIT and experimental data. Experimental pulse-echo A-scan data was collected at locations $A$ and $B$ on the composite microcrack sample (with the transducer centered in the corresponding region) using a $5 \mathrm{MHz}, 1 / 8$ " diameter longitudinal contact transducer. EFIT out-of-plane velocity output was used to calculate pulse-echo A-scan waveforms for locations $A$ and $B$. The EFIT A-scan was created by calculating the Gaussian windowed average over the circular region of the source excitation. An A-scan was also calculated from EFIT data for the case with no microcracks. Figure 5 shows a comparison of EFIT and experimental A-scan data for the microcrack-free IM7/5260 composite sample. The initial excitation has been gated out in time in all A-scan plots shown in this paper. As shown in Figure 5, the EFIT results for the crack-free case compare well with experimental data. Note that we have not included 
material-induced attenuation in the EFIT simulations, thus the EFIT A-scans do not show the material-induced amplitude reduction that appears in the experimental data.

Figure 6 shows EFIT and experimental A-scan data for $A$ and $B$ locations on the microcrack sample. At location $B$ we see extreme crack-induced attenuation in both the EFIT result and in the experimental waveform, yet there are no distinct characteristics in the waveforms to allow for any real measure of the agreement between EFIT and the experimental result. The experimental A-scan data for location $A$, on the other hand, shows distinct peaks corresponding to multiple reflections from the back surface of the sample. The EFIT A-scan shows more severe attenuation and does not contain distinct back wall echo peaks. It is possible that the microCT data used in the EFIT simulation was slightly offset from the transducer location used to collect the experimental A-scan data, in which case, the EFIT simulation may actually contain more extensive microcracking.

These results demonstrate that although real microcrack geometry, location, and distributions have been simulated in EFIT, the attempt to make such direct comparisons with experimental A-scans may require a higher level of precision between experimental data collection location on the sample and the simulated damage region. Additionally, even though the microcrack maps were created using edge finding techniques on high resolution microCT data, it is possible that the resulting maps do not exactly replicate the real sample microcracking. For example, regions of noise in the X-ray CT data may have been designated as cracks in the edge finding/crack mapping algorithm, and/or 'closed' cracks may have been mapped and simulated as fully open cracks (with stress-free boundaries instead of contact). The crack mapping algorithm did visually appear to minimize the potential CT noise issue. Implementing more precise methods to guarantee alignment between the simulated EFIT location and the location of the transducer would be the first step in narrowing in on the cause for the reported discrepancy. Alternatively, we expect that a statistical based comparison between the volume of microcracking and crack-induced attenuation would be a more appropriate approach for comparisons between simulation and experiment. Access to composite samples with varying amounts of microcracking would of course be required for statistical based attenuation comparisons between EFIT and experiment.

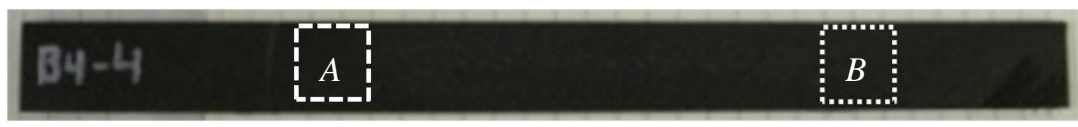

FIGURE 4. Experimental data was collected at two locations, designated $A$ and $B$, on the composite microcrack sample, designated 'B4-4', (with a transducer placed at the centers of the white squares shown in the image). Correspondingly, microCT data for the regions indicated by the white squares was read into the EFIT simulation for comparison to experiment. A-scan comparisons are shown in figures 5-6.

TABLE 1. Single ply material properties for IM7/5260

\begin{tabular}{|c|c|c|c|c|}
\hline $\boldsymbol{\rho}\left(\mathbf{k g} / \mathbf{m}^{\mathbf{3}}\right)$ & $\mathbf{E}_{\mathbf{1}}(\mathbf{G P a})$ & $\mathbf{E}_{\mathbf{2}}(\mathbf{G P a})$ & $\mathbf{E}_{\mathbf{3}}(\mathbf{G P a})$ & $\mathbf{G}_{\mathbf{1 2}}(\mathbf{G P a})$ \\
\hline 1560 & 171.4 & 9.08 & 9.08 & 5.29 \\
\hline $\left.\mathbf{G}_{\mathbf{1 3}} \mathbf{( G P a}\right)$ & $\left.\mathbf{G}_{\mathbf{2 3}} \mathbf{( G P a}\right)$ & $\mathbf{v}_{\mathbf{1 2}}$ & $\mathbf{v}_{\mathbf{1 3}}$ & $\mathbf{v}_{\mathbf{2 3}}$ \\
\hline 5.29 & 2.80 & 0.32 & 0.32 & 0.50 \\
\hline
\end{tabular}




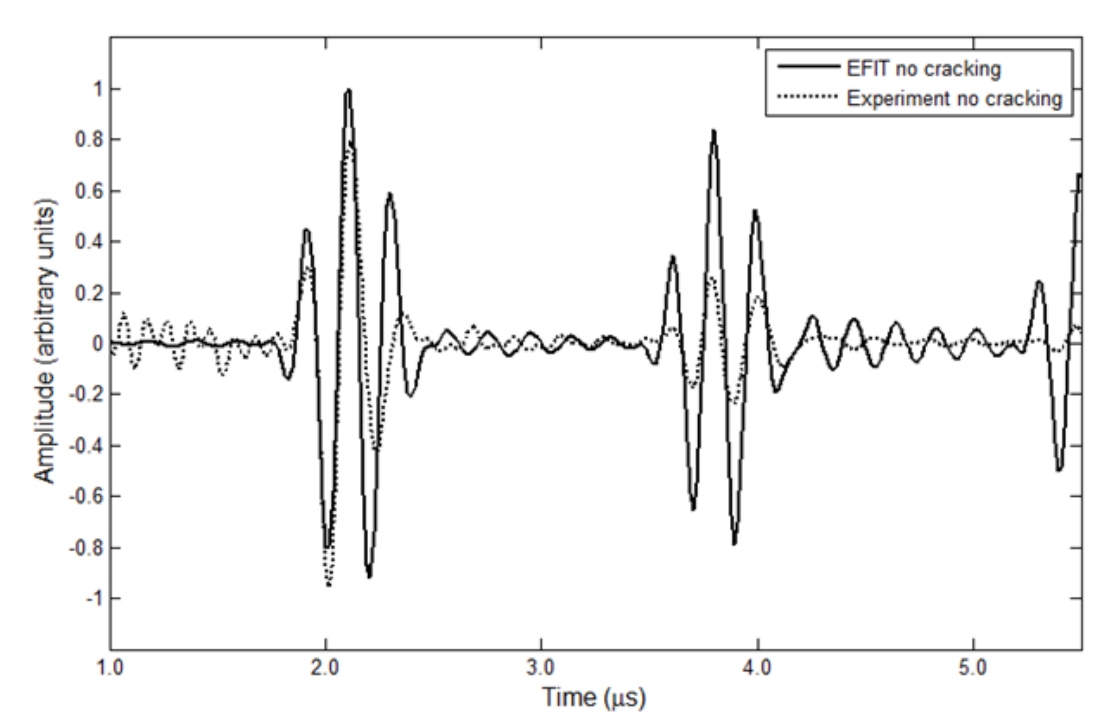

FIGURE 5. Comparison an EFIT A-scan (solid line) and experimental data (dashed line) for the IM7/5260 material for a sample with little to no microcracking. The process of calculating the EFIT A-scan along with a description of expected differences can be found in the text.
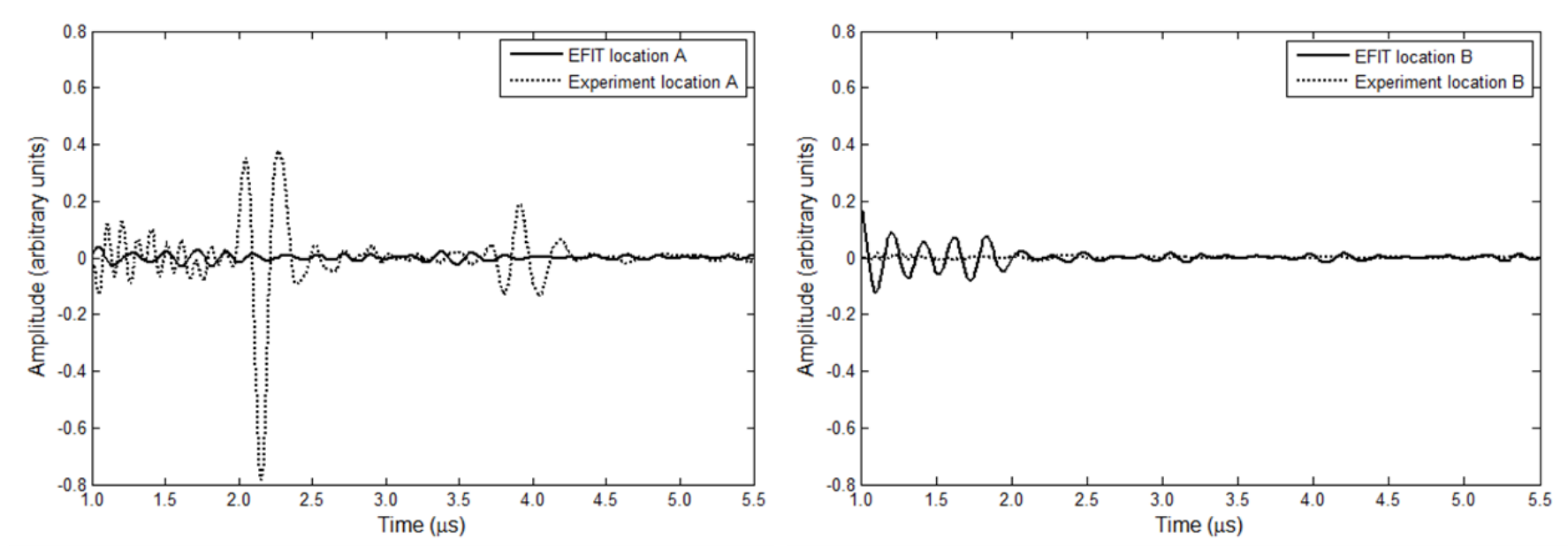

FIGURE 6. Comparison EFIT A-scans (solid line) and experimental data (dashed line) for two different locations on the IM7/5260 sample that contains extensive microcracking. The amplitudes are normalized to the corresponding case shown in figure 5 (e.g., experimental microcrack A-scan amplitude is normalized to the experimental A-scan data for the sample with no microcracking).

\section{CONCLUSION}

We have implemented 3D EFIT simulations to investigate ultrasonic wave interaction with microcracks in composite laminates. Our work demonstrates that EFIT is ideal for incorporating realistic damage types and geometries that are found in composite laminates. Nevertheless, the microcrack A-scan comparisons between EFIT and experimental data are somewhat inconclusive and will require additional investigation, perhaps using samples with less extensive microcracking. Additionally, we plan to move beyond simple comparisons of A-scans to develop a more practical, statistical approach for describing crack induced attenuation. We envision that general trends in crack-induced attenuation versus frequency, using realistic microcrack geometries, could be developed using the 3D EFIT code for composite laminates. 


\section{ACKNOWLEDGEMENTS}

The authors thank Dr. Patrick Johnston of NASA Langley for helpful discussions.

\section{REFERENCES}

1. NASA NESC, http://www.nasa.gov/offices/nesc/home/Feature_CCM.html, accessed $06 / 13 / 2012$

2. I. Daniel and O. Ishai, Engineering Mechanics of Composite Materials, Oxford University Press, New York (2006), pp. 112-113.

3. W.J. Staszewski, C. Boller, and G.R. Tomlinson (Eds.), Health Monitoring of Aerospace Structures, John Wiley \& Sons, West Sussex (2004), pp. 54-59.

4. Hodgkinson, J.M. (Ed.), Mechanical Testing of Advanced Fibre Composites, CRC Press, Boca Raton (2010), pp. 175-176.

5. M. Seale and E. Madaras, "Lamb Wave Evaluation of the Effects of ThermalMechanical Aging on Composite Stiffness", Journal of Composite Materials, Vol 34 (2000), pp. 27-38.

6. C. Leckey, M. Rogge, C. Miller and M. Hinders, "Multiple-mode Lamb Wave Scattering using 3D Elastodynamic Finite Integration Technique", Ultrasonics, Vol 52 (2012), pp. 193-207.

7. C. Leckey and M. Hinders, "3D Modeling of Ultrasonic Wave Interaction with Disbonds and Weak Bonds", Review of Progress in QNDE”, Vol 31 (2012) pp. 111117.

8. F. Fellinger and K. Langenberg, "Numerical techniques for elastic wave propagation and scattering", Proceedings of IUTAM Symposium (1990), pp. 81-86.

9. R. Marklein, R. Barmann, and K.J. Langenberg, "The Ultrasonic Modeling Code EFIT as Applied to Inhomogeneous Dissipative and Anisotropic Media”, Review of Progress in QNDE”, Vol 14 (1995) pp. 251-258.

10. F. Schubert, A. Peiffer, and B. Kohler, "The Elastodynamic Finite Integration Technique for Waves in Cylindrical Geometries", Journal of the Acoustical Society of America, Vol 104 (1998), pp.2604-2614.

11. S. Halkjaer, "Elastic wave propagation in anisotropic inhomogeneous materials", Ph.D. thesis, Technical University of Denmark (2000).

12. C. Leckey, M. Rogge, and F. Parker, "Simulating Three-dimensional ultrasonic wave interaction with realistic damage in anisotropic composites", Journal of the Acoustical Society of America, in press (2012)

13. M. Seale and E. Madaras, "Use of guided acoustic waves to assess the effects of thermal-mechanical cycling on composite stiffness", Technical Report, NASA TM2000-210628 (2000).

14. G. Schoeppner and S. Abrate, "Delamination threshold loads for low velocity impact on composite laminates", Composites: Part A, Vol 31 (2000), pp. 903-915. 